\title{
Search for Wide Double Stars in the Guide Star Catalog I. All Sky Properties of the Catalog
}

\author{
P.L. BERNACCA ${ }^{1}$, B. BUCCIARELLI ${ }^{2,3}$, M.G. LATTANZI ${ }^{2,4}$, \\ R. MORBIDELLI ${ }^{2}$, R. PANNUNZIO ${ }^{2}$, A. SPAGNA ${ }^{2}$, \\ M. SARASSO ${ }^{2}, \&$ A. VOLPICELLI ${ }^{2}$ \\ (1) Asiago Astrophysical Observatory, Italy \\ (2) Torino Astronomical Observatory, Italy \\ (3) Space Telescope Science Institute, USA \\ (4) ESA, Space Science Division, at STScI, USA
}

\begin{abstract}
The immediate object of this effort is the first systematic investigation of the guide star catalog as a new source list for the discovery of possible new visual binary stars. In this first paper of the series we detail on the way used to establish the properties of the catalog, i.e. techniques adopted to measure its completeness as a function of angular separation, magnitude of the primary, and magnitude differences among the components. We have employed the CCDM catalog as the source list of "certified" visual double and multiple stars. The CCDM stars were first matched (rediscovered) in the Guide Star Catalog and then used to build sufficient statistics to establish the relations among the parameters above. These calibrations are essential to properly correct the two- and three-point angular correlation functions, which are our fundamental tools for establishing lists of new probable visual binary and multiple stars.
\end{abstract}

\section{INTRODUCTION}

The angular distribution of stars down to a specified magnitude limit can be analyzed for the detection of gravitationally bound wide double and multiple stars (separations of the order of $0.1 \mathrm{pc}$ ), which are important as probes of Galactic dynamics and disk dark matter. Star catalogs in general and double star catalogs in particular are the source material for such statistical detection of physical pairs. Identification of binary stars is also crucial for photometric and spectroscopic follow-up work.

The task of filtering the physical systems among the objects listed is quite difficult, because source catalogs are usually very inhomogeneous (data taken by different observers with different instruments and detectors), and are not complete. This limits the use of the data and biases the results as selection effects, usually unavoidable (and sometimes unknown), are introduced.

The ideal situation would be a catalog entirely derived from a consistent set of observational material, of known selection criteria and completeness to, for example, a specified magnitude limit. The Guide Star Catalog (GSC - Version 1 of June 1, 1989) is an all-sky survey constructed from digitized Schmidt plates. The detection and cataloging of $2 \times 10^{7}$ objects required the application of automatic selection and classification procedures. Once established and thoroughly tested (Lasker et al. 1990), those procedures remained practically the same for every plate used in the effort. Thus a catalog like the GSC should be a good source list for an all-sky systematic search of wide binaries. However, the situation is not as good as it might sound. As the GSC was created by the STScI for supplying guide stars to accurately point the HST, the goal was not 
that of obtaining a complete, pure sample of stars to a fixed limiting magnitude. Magnitudes range from $6^{\text {th }}$ to $15^{\text {th }}$ but the limits vary with direction on the sky. To make things worse, the plate material is different for the two hemispheres, with the short exposure (20 $\mathrm{min})$ 'Quick-V' plates for $\delta>0$, the SERC-J plates in the South (60 min exposure or more), and the $4^{m}$ exposure SERC-V plates in a strip around the galactic equator (Lasker et al. 1990, Table 3). A further complication is the dependence of image properties on plate position.

In practice, the completeness of the GSC varies with galactic coordinates. For example it is on the average complete around the North galactic pole region (Garnavich 1991), but in general caution must be exercised in interpreting star counts derived from the GSC (Jenkner et al. 1990). Also, before assessment can be made of the quality of the stellar angular correlation functions derived from this catalog, its resolution limit must be firmly established, i.e. the angular separation at which the catalog is complete (all pairs retained), as a function of magnitude.

In this contribution we present first results on some of the GSC properties. In particular, we give the results of the first direct attempt to determine the GSC resolution limit in the Southern hemisphere.

\section{REFERENCE DATA AND CALIBRATION PROCEDURE}

There are basically two types of reference data which can be used to test the basic properties of the GSC, i.e. general double star catalogs and complete double star catalogs (plate catalogs) restricted to small regions of the sky and based on the same plate material used for the GSC determinations, for which the resolution limit is determined with high accuracy (see Garnavich 1991 for the description of such catalogs in the North).

We have used the Catalogue de Composantes d'étoiles Doubles et Multiples (CCDM) (Dommanget 1983), as the source list of 'certified' visual doubles. For this investigation, and to minimize the errors during the cross-matching, we reduced the number of the CCDM entries by using only those systems whose positional precision (for the primary components, at least) is given to $1^{\prime \prime}$ or better and with the magnitude of the primary fainter than $m_{v}=7$. This leaves 12,374 systems over the entire sky. Presently, we have run the cross-matching for the first 12 hours of GSC right ascensions. This reduces the comparison sample to 5,185 doubles.

Figure 1 shows the distribution of the separation of these stars. It is clear that the resolution of our comparison catalog is well above that expected for deep Schmidt plates $\left(\simeq 4^{\prime \prime}\right)$ and, then, suitable for the determination of the GSC resolution limit. Figure 2 shows the magnitude distribution of the CCDM primaries. As expected, the CCDM is a fairly bright catalog (the average magnitude is around $m_{v}=9.3$ ). However, as we are only interested in relative comparisons, we still have statistically meaningful samples down to $m_{v}=12$. Finally, the histogram in Figure 3 represents the $\Delta m$ [in the sense $m_{v}$ (secondary) $-m_{v}$ (primary)] distribution. It covers a more than adequate range.

In order to 'rediscover' the CCDM stars in the Guide Star Catalog we have defined a multiparameter matching space with the intent to isolate the potential GSC candidates according to the prescription of the cluster analysis (see Kauf- 
man \& Rousseeuw 1990). As parameters we included the differences in position $(\Delta P)$ between the primary component $A$ in the CCDM and the candidate primary $a$ in the GSC, the difference in separation $\left(\Delta \rho=\rho_{C C D M}-\rho_{G S C}\right)$, the difference in position angle $(\Delta \vartheta$, expressed as linear displacement of the two separation vectors - one for the CCDM and one for the GSC), and finally the magnitude differences $\Delta m_{A}$ and $\Delta m_{B}$ between the two primaries and the two secondaries. A standardized 5-dimensional euclidean distance is computed for each GSC candidate around a given CCDM star (the size of the search window around each CCDM target was usually $270^{\prime \prime}$ in radius). Standardization is obtained by dividing each quantity by the corresponding estimated error. The adopted approximate values were $\sigma_{\Delta P}=1^{\prime \prime} .3, \sigma_{\Delta \rho}=0^{\prime \prime} .8, \sigma_{\Delta \vartheta}=1^{\prime \prime} .5, \sigma_{\Delta m_{A}}=$ $0 " 7$, and $\sigma_{\Delta m_{B}}=0 " 19$. Thus, the most probable GSC double, i.e. the one with the shortest distance, is considered to match its CCDM counterpart.

Of the 5,035 CCDM stars with separation less than $60^{\prime \prime}, 979$ were found in the GSC. About $78 \%$ of these stars (767) are in the North.

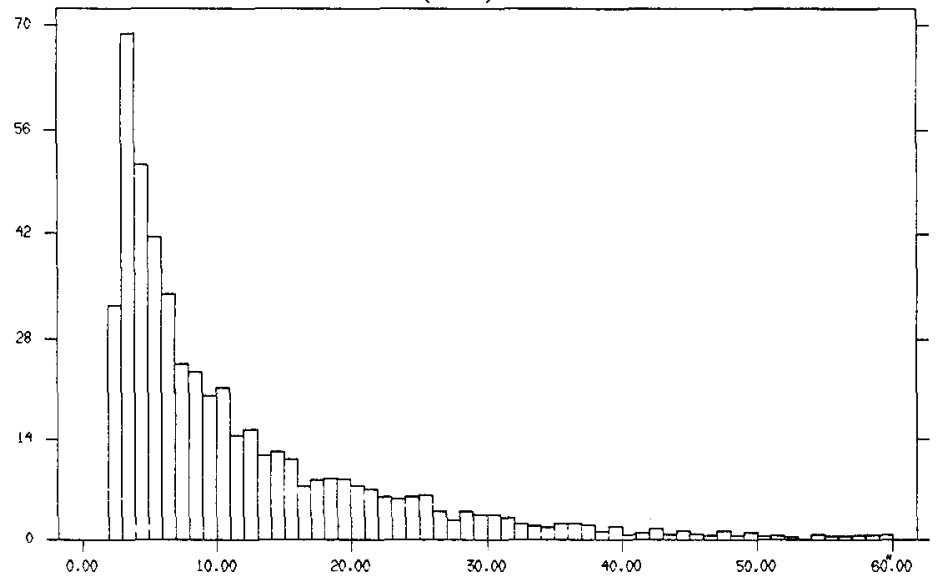

FIGURE 1. Distribution of the separations of the CCDM pairs included in our reference list (see text).

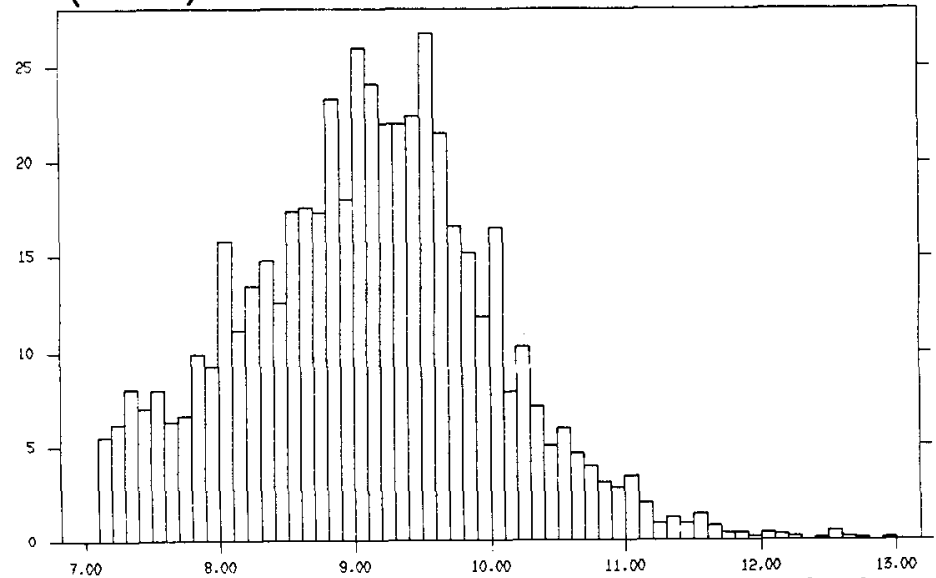

FIGURE 2. Magnitude distribution of the primary components for the same sample of CCDM pairs. 


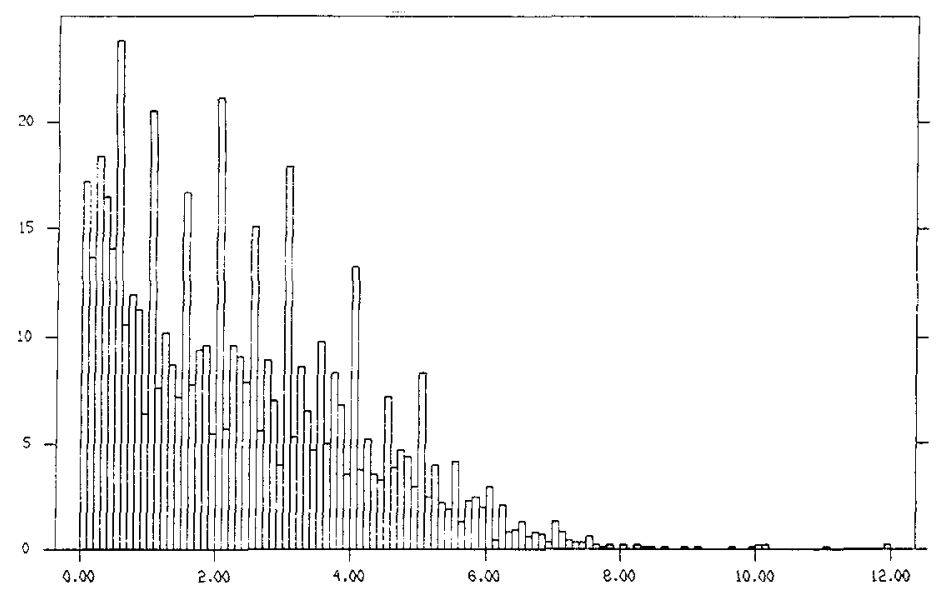

FIGURE 3. Distribution of the magnitude difference between the primary and the secondary components for the same sample of pairs as Figures 1 and 2.

The quality of our matching procedure is quite satisfactory. The distribution of $\Delta P$ shows that the great majority of the data points is below the $2^{\prime \prime}$ limit which is consistent with the $\sigma_{\Delta P}$ quoted above. The southern distribution of $\Delta P$ appears somewhat flatter. This is not surprising as image centering errors depend on image diameter and the southern exposures are on average $3-4$ times deeper than the northern ones. Also, the second moment of the distribution of $\Delta \rho$ is consistent with the value of $\sigma_{\Delta \rho}$ adopted for the matching.

\section{RESULTS}

The fraction of pairs successfully recovered by the GSC as a function of separation is shown in Figure 4 for the northern sky (full line). The cut-off $\left(\rho_{c}\right)$ at $10^{\prime \prime}$ indicates that the GSC cannot resolve pairs closer than that limit. As the bright stars (around the 9th magnitude) dominate in the CCDM, it could be that $\rho_{c}$ is not representative of the GSC. However, Garnavich (1991), using his plate catalogs as comparison (complete to $\rho \simeq 4$ " in the magnitude range $V=8$ 14), came to the same conclusion noticing that "Even for the faintest primaries the GSC is unlikely to resolve and correctly class pairs of stars closer than $10^{\prime \prime}$. The resolution limit, as defined in the previous section, appears to be at $\rho_{l i m} \geq$ 25 " (the fraction of recovered pairs settles at around $90 \%$ ).

The plot for the southern sky is the dashed line in Figure 4. Somewhat surprisingly, the cut-off appears to be again around $10^{\prime \prime}$, as for the North. However, now the pairs are recovered in an irregular fashion and much more 'slowly' than before. Also, there is no clear evidence for the onset of $\rho_{\text {lim }}$, although we can conclude that $\rho_{\text {lim }}$ for the southern sky is larger than $30^{\prime \prime}$, if we assume that there is one.

Why are the two plots of Figure 4 so different? One explanation could be that the two surveys that went into the GSC are intrinsically very different. Indeed, the southern material is not just deeper plates. Emulsion, telescope, 


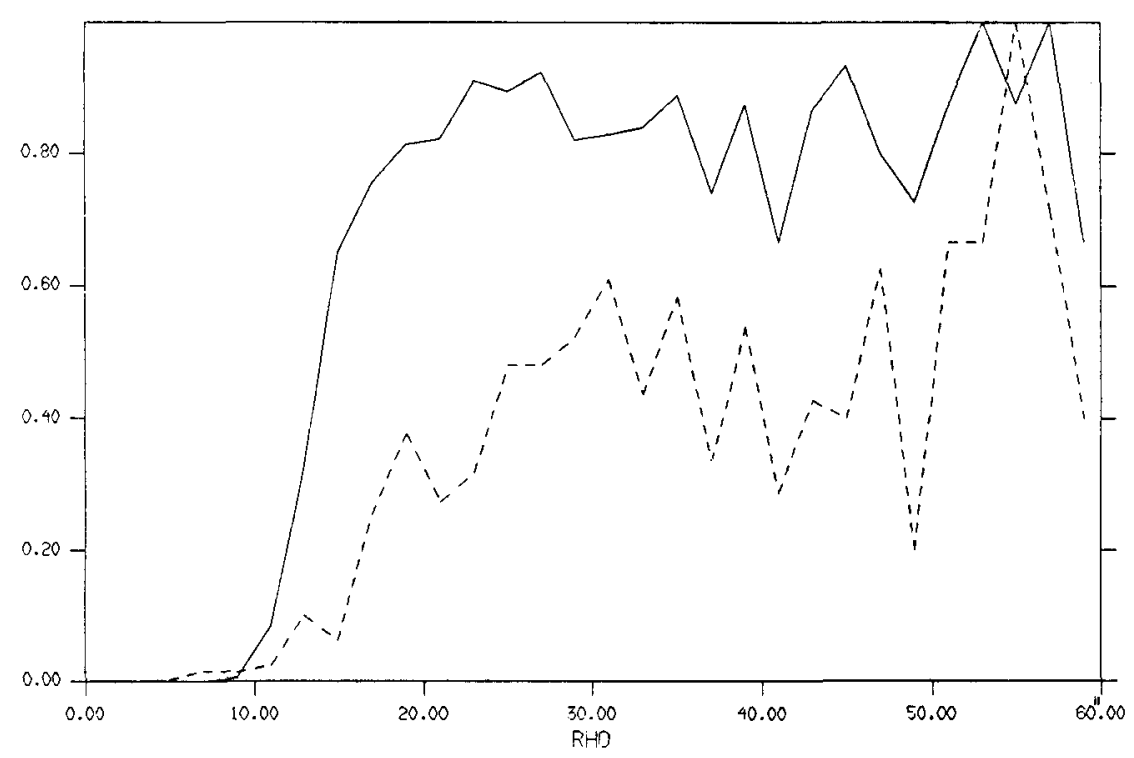

FIGURE 4. Solid line: fraction of CCDM pairs recovered in the GSC as a function of separation (Northern sky). Dashed line: Same distribution for the Southern sky.

passband, and processing differences are such that the image properties of the two surveys are markedly different. However, a word of caution is required at this point. In the previous section we said that only 212 CCDM pairs $(22 \%$ of the whole sample) were rediscovered in the southern section of the GSC. Also, Figure 1 shows that the distribution of the separation in the CCDM peaks at relatively small values, thus Figure 4 could be the result of large statistical fluctuations. We prefer to wait until the CCDM-GSC match is completed before deriving any statistically robust conclusion.

All the plots and the statistical calculations were done using the package DIRA (Astronet Data Base Group 1992).

\section{REFERENCES}

Astronet Data Base Group, 1992, Distributed Information Retrieval for Astronomical files, Version 2, User Manual

Dommanget, J. 1983, Un Catalogue de Composantes d'etoiles Doubles et Multiples (CCDM), CDS Inform. Bull. 42, 83

Garnavich, P. 1991, Ph.D. thesis, University of Washington

Jenkner, H., Lasker, B.M., Sturch, C.R., McLean, B.J., Shara, M.M., \& Russell, J.L. $1990, A J, 99,2082$

Kaufman, L. \& Rousseeuw, P. 1990, Finding Groups in Data, (New York, John Wiley \& Sons)

Lasker, B.M., Sturch, C.R., McLean, B.J., Russell, J.L., Jenkner, H., \& Shara, M.M. $1990, A J, 99,2019$ 